\title{
Visual Summarization of Lecture Video Segments for Enhanced Navigation
}

\author{
Mohammad Rajiur Rahman \\ mrahman13@uh.edu \\ University of Houston \\ Houston, TX
}

\author{
Jaspal Subhlok \\ jaspal@uh.edu \\ University of Houston \\ Houston, TX
}

\author{
Shishir K. Shah \\ sshah5@uh.edu \\ University of Houston \\ Houston, TX
}

\begin{abstract}
Lecture videos are an increasingly important learning resource for higher education. However, the challenge of quickly finding the content of interest in a lecture video is an important limitation of this format. This paper introduces visual summarization of lecture video segments to enhance navigation. A lecture video is divided into segments based on the frame-to-frame similarity of content. The user navigates the lecture video content by viewing a single frame visual and textual summary of each segment. The paper presents a novel methodology to generate the visual summary of a lecture video segment by computing similarities between images extracted from the segment and employing a graph-based algorithm to identify the subset of most representative images. The results from this research are integrated into a real-world lecture video management portal called Videopoints. To collect ground truth for evaluation, a survey was conducted where multiple users manually provided visual summaries for 40 lecture video segments selected from a diverse set of courses. The users also stated whether any images were not selected for the summary because they were similar to other selected images. The graph based algorithm for identifying summary images achieves 78\% precision and 72\% F1-measure with frequently selected images as the ground truth, and $94 \%$ precision and 72\% F1-measure with the union of all user selected images as the ground truth. For $98 \%$ of algorithm selected visual summary images, at least one user also selected that image for their summary or considered it similar to another image they selected. Over $65 \%$ of automatically generated summaries were rated as good or very good by the users on a 4-point scale from poor to very good. Overall, the results establish that the methodology introduced in this paper produces good quality visual summaries that are practically useful for lecture video navigation.
\end{abstract}

\section{KEYWORDS}

lecture video summarization, lecture video navigation, lecture video, educational video, screencast

\section{INTRODUCTION}

Recorded lecture videos are gaining popularity as a core tool for distance learning as well as a supplementary tool for face-to-face learning. Popular commercial lecture video hosting platforms include Echo360[17], Kaltura[30], and Panopto[46]. When classroom lectures are captured on video and made available to students, they make use of them, enjoy using them, and perceive them to be a valuable learning tool $[1,2,6,7,9,11,14,15,28,33,45,48,52,56]$. Lecture videos positively impact traditional lecture based coursework as well as online learning. In situations where videos are provided prior to class, and class time is instead used for active learning, positive effects include increased student retention, learning, and engagement $[8,19,26,32,49]$. Students value videos for allowing them to review at their own pace $[6,15,28,51,52]$ and typically report that their use of videos had a positive impact on grades and overall course satisfaction $[6,11,15,28,33,44,52]$.

This research is conducted in the context of Videopoints project at the University of Houston whose central goal is to ease navigation of lecture videos, making them a companion resource for learning, similar to a textbook. An important limitation in employing lecture videos for learning is the challenge of quickly accessing a video segment that contains the content of interest. Conventional video format inherently lacks non-linear navigation support like indexing and content search. Videopoints overcomes this limitation by partitioning the video into segments discussing a sub-topic in the lecture, and presenting the segments to the users via visual index frames $[55,56]$.

The main goal of the research presented in this paper is to build a visual summary to provide a natural way to connect to a lecture video segment. These visual summaries are employed to index lecture video segments to improve navigation. A visual summary is expected to be more informative for the user than the first frame of a video segment that is often used by default for indexing. The video summary can be used in conjunction with a text summary [31] in a real world system like Videopoints.

The approach taken in this work is based on analyzing the video frames in a lecture video segment, identifying the most relevant images, and synthesizing a new frame that is representative of the visual objects in the video segment. In practice, we only need to analyze the transition frames, which are the video frames where the scene in the video changes based on the RGB values of corresponding pixels $[22,23,43,56]$. We outline the process of generating the visual summary. First, all images on each transition frame are identified. Text portions in a frame are identified with OCR tools and not considered to be images. Next, a "visual distance" between each pair of images is calculated based on low level image features. Finally a subset of most representative images is identified based on the image distance matrix and other factors including the size of the image and the amount of time it is displayed in the video.

Visual summarization is illustrated in Figure 1. There are four transition frames identified within a video segment. A possible visual summary is shown to the right in the figure.

\section{BACKGROUND}

Proposed research has its roots in the Videopoints project. The project developed a lecture video framework illustrated in Figure 2 that is in active use. Various aspects of this work are reported in detail in $[2,3,13,53,54,56,57]$. We briefly describe the Videopoints 

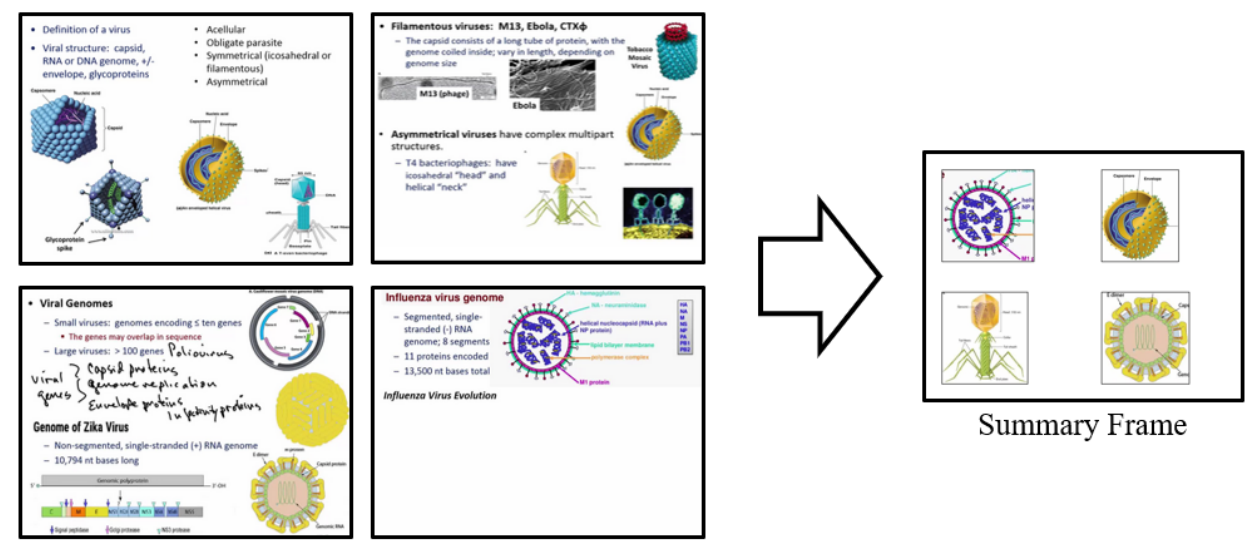

Summary Frame

Transition Points of a Video Segment

Figure 1: Visual Content Summarization

player that encapsulates indexing, search, and captioning. An index panel is situated on the bottom of the player; each index point represents a new topic in the form of a screen-shot of the video at that point of time. Users can navigate different topical segments of the video by clicking on these index frames.

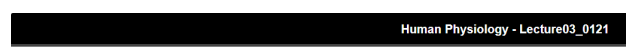

Secretory Pathway
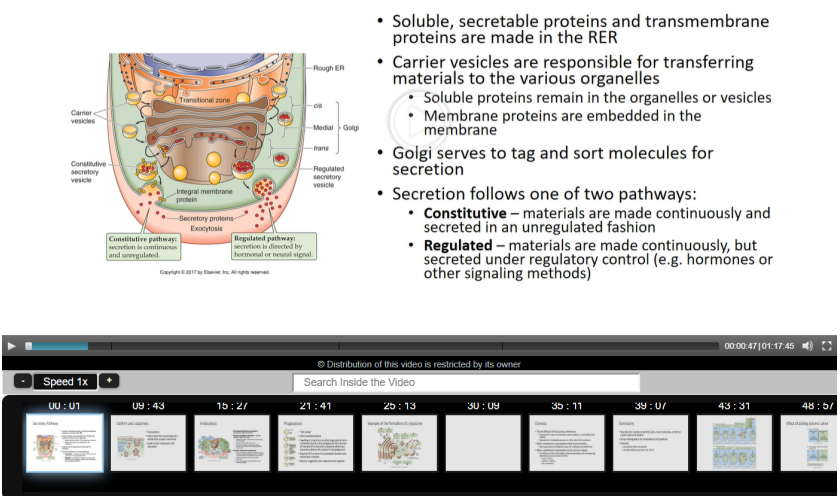

Figure 2: Videopoints Player

This video framework has been deployed at University of Houston and used for coursework across Biology, Chemistry, Computer Science, Geology, Mathematics, and Physics. More than 3,500 students in 73 course offerings were surveyed [55]. Figure 3(a) shows that the students considered access to lecture videos and the player very important and figure 3(b) shows that student users were generally satisfied with the topical indexing feature.

\section{RELATED WORK}

Video summarization is a long-studied problem in multimedia content analysis. Several projects have attempted to summarize videos to facilitate quick retrieval or browsing of large volumes of data generated by video cameras [12, 16, 20, 29, 59]. These approaches try to identify a small set of frames in a video that can convey the overall content of the video, that are then presented to the user. These video summarization techniques have been investigated mostly for video obtained from typical video cameras. The results are not directly applicable to lecture videos or screencasts, which is the main focus of this research.

In recent years, a body of research has been developed to analyze lecture videos. Several contributions focus on finding unique transition frames or minimizing the number of frames of videos across different types of presentations like powerpoint lectures and blackboard handwriting [10, 25, 29, 37, 38]. Recent research has also focused on methods for efficient access to educational videos with automatic identification of keywords from videos or their segments $[31,36]$.

One contribution indirectly related to our work is ViZig [58]. In this work visual content is extracted, classified, and presented with a direct link to the player timeline where it appeared in the video to help non-linear navigation. However, this work does not address visual summarization.

In the context of identifying images relevant for visual summarization, this research leverages existing methods for detecting and matching interest points to establish a measure of visual similarity between images. Several interest point detection algorithms such as Harris corner detector [24] and others such as the HessianLaplace, Laplacian-of-Gaussian, and the Laplacian-of-Gaussian using a Difference-of-Gaussian [35, 39, 41, 42] and numerous features such as SIFT [39], SURF [4], GLOH [18, 34], DAISY [50], and their variants $[5,18,34]$ have been proposed and their usage demonstrated to a range of image analysis applications including image matching problems.

\section{VISUAL CONTENT SUMMARIZATION}

The main technical objective of this paper is to generate a visual summary of a lecture video or screencast, where the content on the screen consists of text and images, that change often but not 


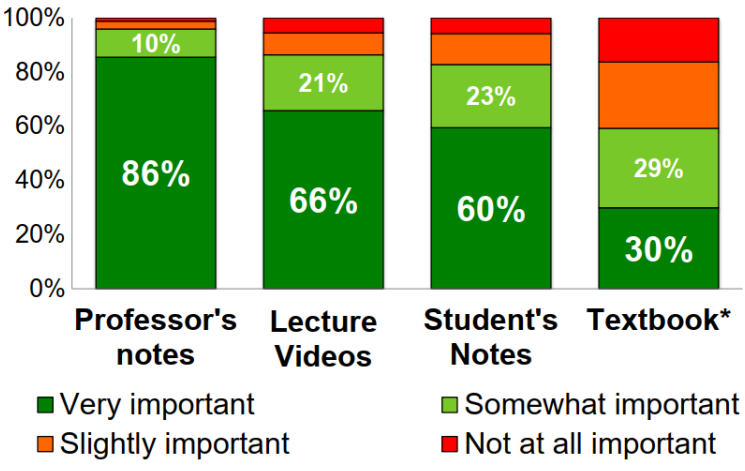

(a) Student ratings of learning resources

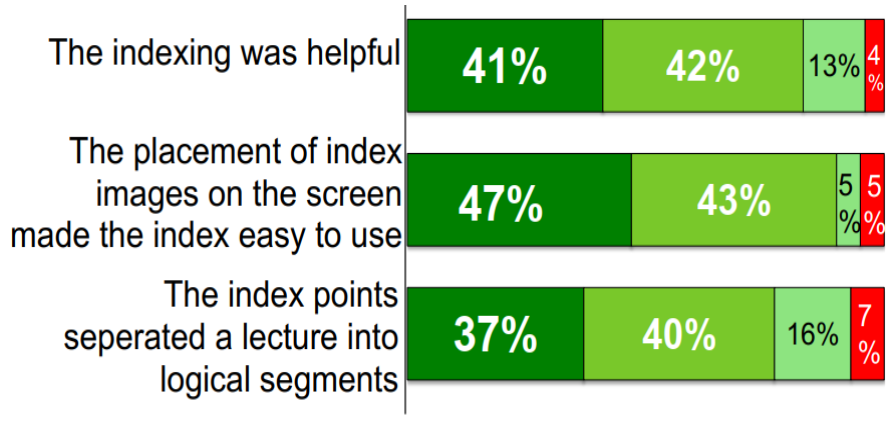

Agree strongly $\quad \square$ Agree $\quad \square$ Agree slightly $\square$ Disagree

(b) Perception of value of indexing

Figure 3: Lecture video as a learning resource and its evaluation

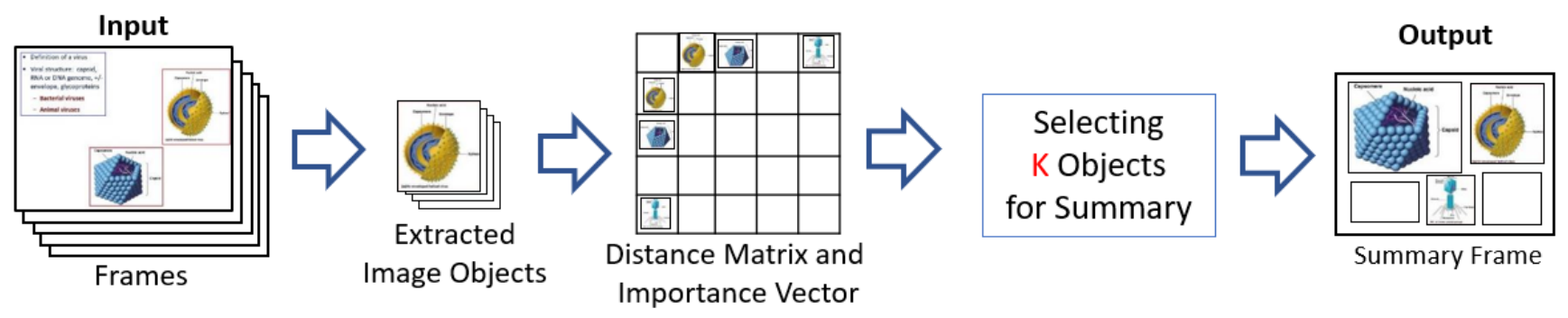

Figure 4: Algorithm Steps

continuously. Specifically, the goal is to identify a subset of the images from the frames of the video segment that best represents the content of the lecture segment. The approach taken for generating the visual summary of a lecture video segment in this paper consists of the following steps:

(1) Extract all images from the frames in the video segment, along with the time for which they were displayed in the video timeline.

(2) Compute the "importance" of each individual image for inclusion in the visual summary.

(3) Compute the "distance matrix" between all pairs of images based on (dis)similarity between the images.

(4) Select the subset of images that best represent the segment based on similarity and importance.

These steps are illustrated in Figure 4. We describe the steps 1-3 in this section which generate an importance vector, and an imageimage distance matrix. The selection of representative images from a distance matrix is detailed in section 5 . The final selected images are placed in a single frame on uniformly sized cells. A more visually appealing arrangement is possible but beyond the scope of this work. An example set of lecture video frames and the corresponding visual summary is illustrated in Figure 1.

\subsection{Extracting Images from a Lecture Video Segment}

A lecture video or screencast typically consists of a small number of unique video frames, each displayed from a few seconds to several minutes. The content of the video frame is often a Powerpoint viewgraph, but it can be an image from the web or elsewhere. Following are the steps to extract the image objects in the frames of the segment.

(1) Identify transition frames: The first step in this analysis is to identify transition frames where the scene in the video changes significantly based on the RGB values of the corresponding pixels. Figure 1 shows the 4 transition frames in an example lecture video segment.

(2) Remove text regions: The next step is to remove text regions from the transition frames. This is to prevent blocks of text being identified as images. We employ an OCR engine for this purpose. OCR extracted text regions are grouped together in a block of text, if they are close to each other.

(3) Identify image regions: At this stage, the transition frames consist of images and empty space. We employ a simple technique to identify image regions that proceeds as follows. A transition frame is scanned for pixel changes with a sliding window protocol. Bounding boxes enclosing image objects are identified as regions surrounded by a border with no visual content. 


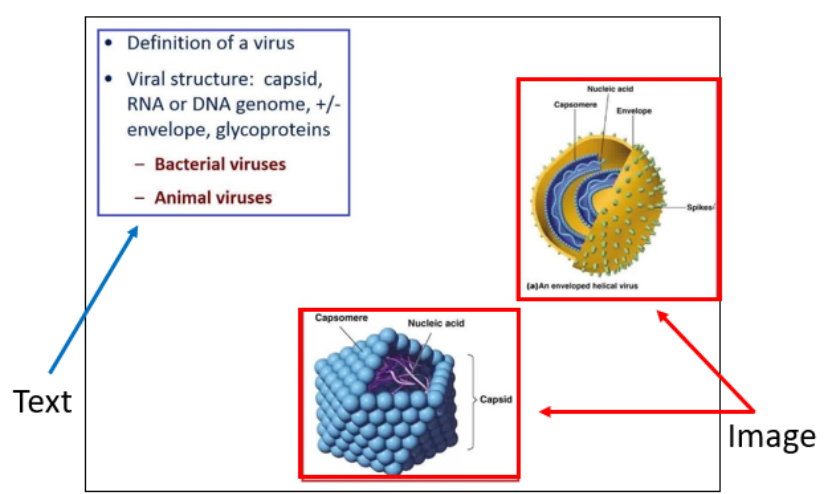

Figure 5: Extracting Images from a Lecture Video Segment

A transition frame with boxes containing image objects and a text region are illustrated in Figure 5. This work leverages the framework developed for identifying transition frames and extracting text with OCR for content based indexing [54, 57].

\subsection{Image Importance}

Desirability of an image to be included in a visual summary is an independent consideration from similarity to other images. This importance of an image depends on a number of factors including the size of image, information density in the image, and the duration for which the image is visible in the lecture video. The information density is captured by number of keypoints in unit area. We have developed a heuristic definition of importance as follows. We normalize these features for all images in the segment and then calculate the importance using following equation:

$$
\text { Importance }=\text { Size } * \text { InfoDensity } * \text { Duration }
$$

\subsection{Image Distance Matrix}

An important consideration in deciding whether an image should be included in a visual summary is to quantify how similar (or different) it is to other images. In this work we calculate the similarity between each pair of extracted image objects and create a distance matrix, where

$$
\text { Distance }=1-\text { Similarity }
$$

Computing a measure of similarity between two images is a well studied problem. There are many metrics and algorithms to measure image similarity. Global measures include holistic image properties such as color and texture computed from the entire image or parts of an image, often represented as histograms. In contrast, local measures rely on identifying parts or points within an image that are unique to an image or objects within an image, which in turn can be used in computing similarity between images. Finding similarity of visual objects in a lecture video is a unique problem as the images are typically synthetically created and do not represent any real world objects. Images often contain illustrations like diagrams, cartoons, charts, and graphs. The images often have a specific meaning in a particular domain only. Images tend to have geometrical shapes. Pairs of images, where one image is a rotated, scaled, or cropped version of another, are common in a lecture video segment. It is also common that one image is simply another image with additional visual content.

Based on above considerations and our practical experience, we chose to focus on local features that are invariant to geometric transformations. We chose to use SIFT [39] to extract local interest points (keypoints) and the corresponding feature descriptors from an image to be used for matching interest points between images. To compute a measure of similarity between two images, we measure:

- KeypointsScore. The percentage of unique keypoints that match between the pair of images; and

- TransformationScore. The degree to which one image is a geometric transformation of the other.

The percentage of keypoints matched provides an indication of local similarities between two images. We further compute an affine transformation based on matched keypoints. The second image is transformed and aligned with the first image using the computed transformation and a pixel-wise normalized difference is measured to provide an indication of the global similarity between two images. The final similarity score is simply the average of these two measures, given as:

$$
\text { Similarity }=\operatorname{avg}(\text { KeypointsScore }, \text { TransformationScore })
$$

\section{SELECTION OF REPRESENTATIVE IMAGES}

In section 4 we described the process of obtaining the set of images in a lecture video segment, an importance value for each image, and a distance matrix that captures the dissimlarity between pairs of images. In this section we discuss the mechanism of identifying a small subset of those images that best represent the content of the lecture video segment. We first present a theoretical formulation of the problem and then present practical algorithms to solve it.

\subsection{Problem Description}

Suppose a lecture video segment has $n$ images (or visual object), $V_{1}, V_{2}, V_{3}, V_{4}, \ldots, V_{n}$. The goal is to construct a visual summary consisting of $m$ representative images, $R_{1}, R_{2}, R_{3}, R_{4}, \ldots R_{m}$ where $m<n$. An nxn Distance matrix is available where Distance $_{i j}$ captures the visual difference between images $V_{i}$ and $V_{j}$. A vector Importance of size $n$ is provided where Importance ${ }_{i}$ captures the importance of the corresponding image $V_{i}$.

For identifying representative images for the summary, we apply two considerations: i) minimize the distance between each image not in the summary to the closest representative image in the summary, and ii) prioritize images that have more importance. Quantitatively our optimality criterion is to identify a set of representative images for which the maximum of Distance ir $*$ Importance $_{i}$ over all images is minimized, where Distance ir $_{r}$ is the distance between image $V_{i}$ and the image $V_{r}$ in the summary that is closest to $V_{i}$.

\subsection{Optimal representative image selection}

We informally show that the problem of optimally selecting representative images as stated in this section is NP-hard.

Consider a highly simplified version of the problem where i) all images are of equal importance and ii) the visual distance between any pair of images is 0 (identical) or infinite (no similarity). We can think of this version of the problem as a graph problem where 
each of the images $V_{1}, V_{2}, V_{3}, \ldots V_{n}$ is a graph node. There is an edge between the nodes if the corresponding visual distance between the images is 0 , and no edge if the corresponding distance is infinite. In this scenario, the problem of finding an optimal summary of $m$ representative images translates to finding $m$ graph nodes, such that all other graph nodes are adjacent to one of the nodes in this set. This is the exact formulation of the problem of finding a dominating set of a graph which is known to be NP-hard [21]. Hence the problem of identifying representative images as detailed in this section is NP-hard.

\subsection{Graph based algorithm}

While the general problem of finding the optimal visual summary is NP-hard, the real world instance of interest to us is not as daunting as the number of images in a segment is typically not that large. In most instances, even an exponential algorithm that examines all combinations of images as candidates for the visual summary can be run within a few minutes on a desktop. However, this is not a practical approach above a certain size and visual complexity of a lecture video segment.

We introduce an efficient heuristic algorithm for identifying a good set of representative images that proceeds as follows. Initially the visual summary consists of all images in the segment. In the following step, the cost of removing each image from the summary is computed.

The cost $\cos _{k}$ of removing image $V_{k}$ is computed as follows:

$$
\operatorname{cost}_{k}=I_{k} * D_{k, p}
$$

where $V_{p}$ is the node in the summary that has the least distance (or is most similar to) $V_{k}, I_{k}$ is the importance of image $V_{k}$ and $D_{k, p}$ is the distance between $V_{k}$ and $V_{p}$. The node with the lowest cost is removed. This step is repeated until the desired $m$ nodes are left in the summary. The complexity of this heuristic algorithm is $O\left(n^{3}\right)$ for a segment with $n$ images ${ }^{1}$.

For the data set employed for experiments in this paper, we verified with a brute force approach that the results from the heuristic algorithm nearly always matched the optimal results.

\section{EVALUATION}

The results of visual summarization were tested and evaluated in the context of Videopoints portal for lecture videos that is widely used at the University of Houston. Results are presented for the graph based algorithm presented in this paper, as well as the Kmedoid clustering based algorithm. We describe the process of ground truth collection and the metrics used to evaluate the quality of the summaries generated by our algorithms.

\subsection{Ground truth collection}

Ground truth was collected with a survey of users of the Videopoints system.

Dataset. We selected 40 segments from 4 subjects taught at the University of Houston. The subject areas, in decreasing order of

\footnotetext{
${ }^{1}$ Strictly speaking, the complexity of the algorithm as stated for illustration in the paper would be $O\left(n^{4}\right)$ but a trivial change that records the closest node in summary set for all other nodes would make it $O\left(n^{3}\right)$.
}

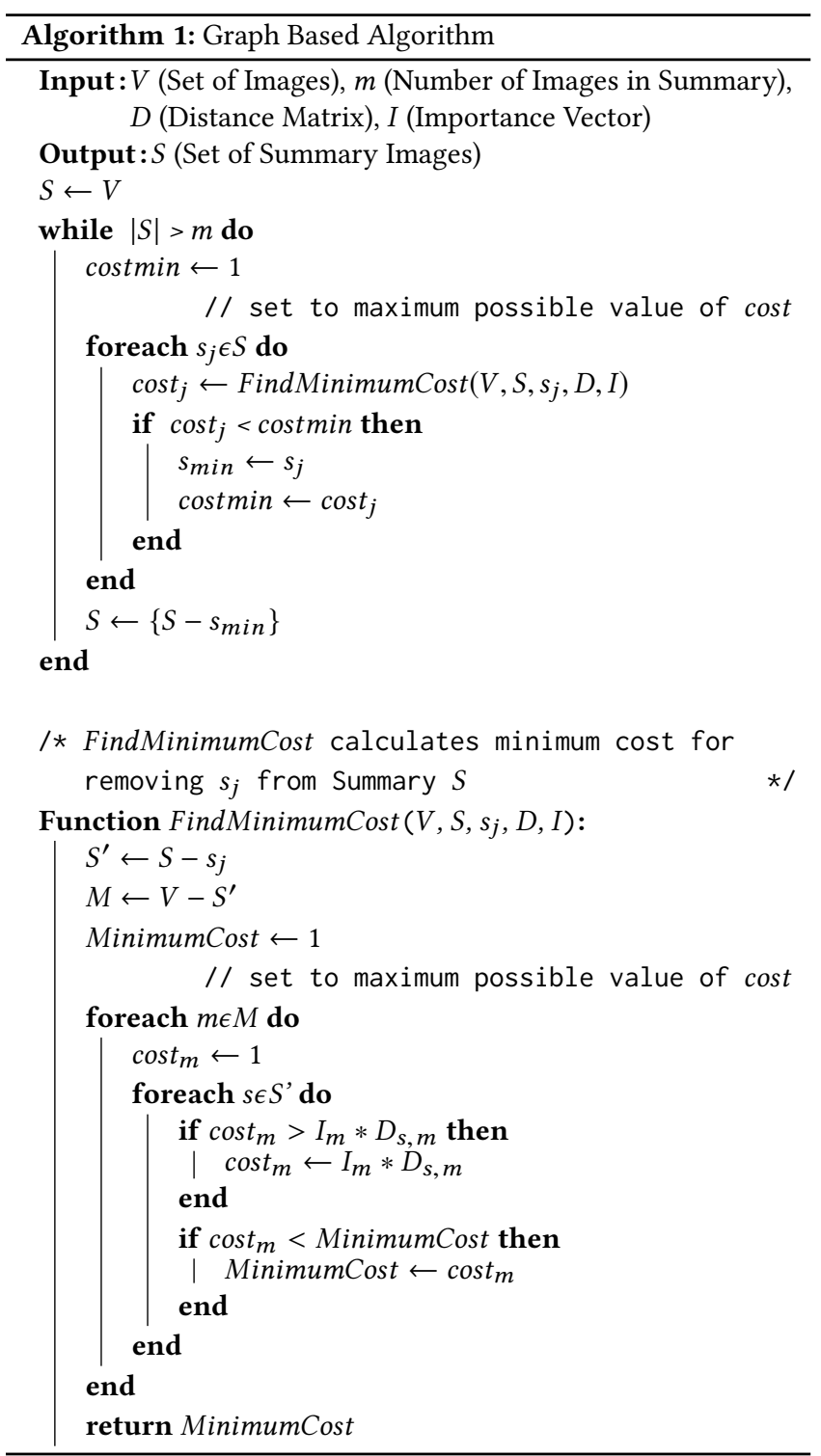

the number of selected segments, are Biology, Geoscience, Computer Science and Chemistry. The segments were approximately 15 minutes long on average, and contained approximately 12 images on average. Segments with 5 or fewer images were not selected for evaluation. For our experiments, the algorithms were configured to provide a visual summary of up to 4 images, and the task of selecting a 4 image summary is trivial or relatively easy in these cases. More details on the data set are listed in Table 1.

Survey. We developed a web-based survey tool and process to collect ground truth that proceeds as follows:

(1) A survey participant is first shown all distinct images extracted from a video segment, and asked to select a set of 4 images that best represent the segment. This is illustrated with an example in Figure 6. 
Following are the frames in a video segment:
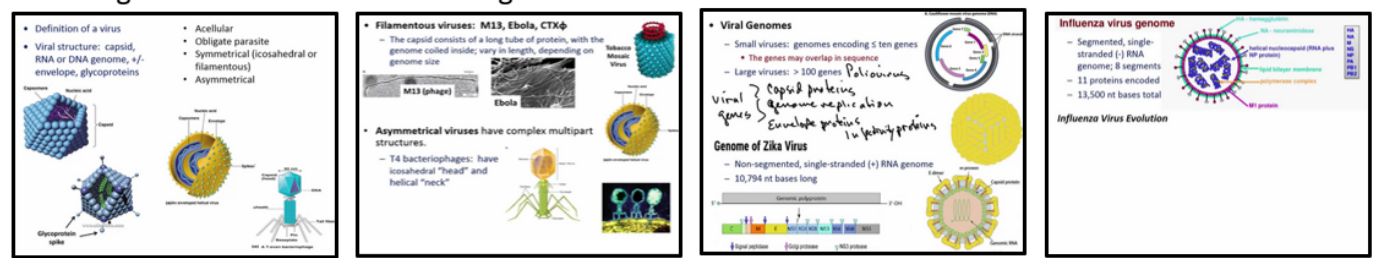

In your view, which subset of images below best capture the content of this segment? Select up to four:
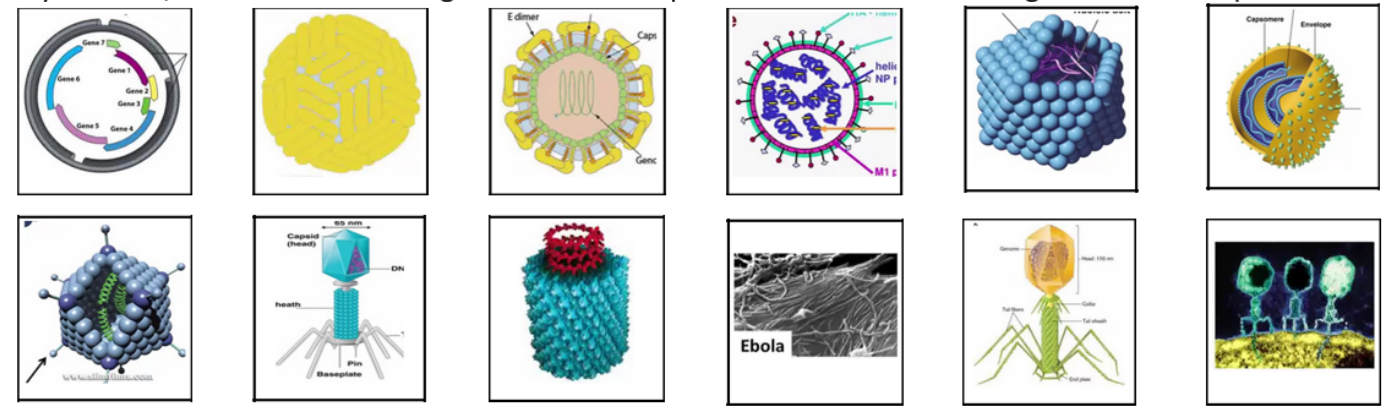

Figure 6: Interface to identify images for the ground truth visual summary

Table 1: Statistics on video segments for evaluation

\begin{tabular}{|l|c|c|}
\hline & Duration (mins) & Total Images \\
\hline Min & 2.87 & 6 \\
\hline Max & 36.03 & 30 \\
\hline Average & 14.99 & 12.28 \\
\hline Median & 14.04 & 11 \\
\hline
\end{tabular}

(2) Next, for every image a participant did not select for the visual summary, they are asked if the primary reason was i) it was not as important as selected images, or ii) it was similar to one of the selected images. An example is shown in Figure 7.

(3) Finally the participant is presented with the images automatically selected by our algorithm and asked to judge the quality of this summary on a 4 points scale with 1 as "Very Good" and 4 as "Poor". In the same step, they are asked to rate their familiarity with the content of the lecture video on a 4 point scale, with 1 as "Very Familiar" and 4 as "Not at all Familiar". An example is shown in Figure 8.

Participants. Students and instructors were invited to participate in the survey. Since domain knowledge in the subject area is an important factor, we focused on recruiting instructors and students in the subject areas of the survey. The participants self reported areas that they were familiar with, and were assigned segments in those areas. The survey had 30 participants. Each segment was ranked by 5.75 participants on average, with 6 being the maximum and 3 being the minimum for any segment.

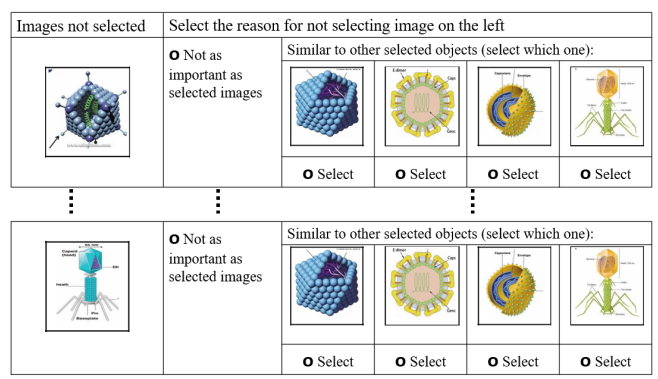

Figure 7: Interface to identify the reasons for not selecting specific images for the visual summary (truncated to show only 4 images)

Visual Summary

generated from the images you selected
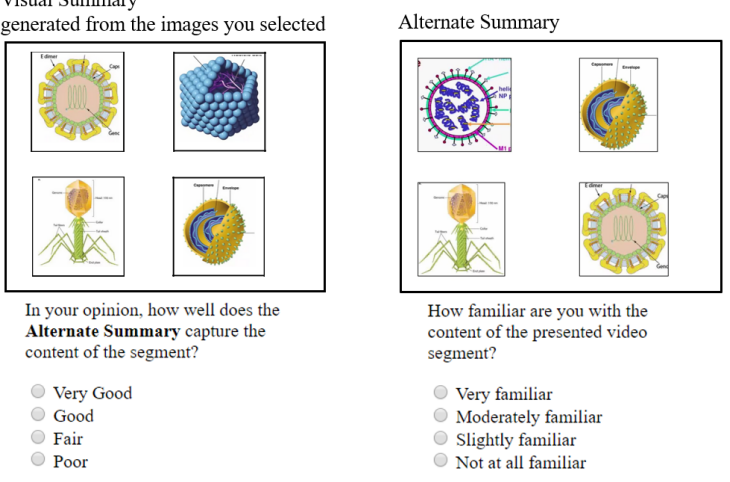

Figure 8: Interface to rate the quality of an automatically generated summary and to rate user familiarity with the subject area 


\subsection{Evaluation Methodology}

Evaluation of a visual summarization algorithm is challenging for a number of reasons:

(1) Multiple images can express the same concept and hence a user may consider two or more images equally representative of a subtopic in a video segment. To capture this aspect, we allow users to specify such perceived similarity in the ground truth collection survey.

(2) There may be no consensus on ground truth. Users often differ significantly on their assessment of the best set of images that summarize a video segment.

(3) Different sets of images may represent the content of a video segment equally well, even if they have few images, if any, in common.

We have developed an evaluation methodology to address these factors. We outline the salient aspects of our approach to evaluation:

Individual images selected by participants: During ground truth collection, different survey participants pick different images for a visual summary. We perform evaluation on a subset of images that are:

- Selected most often by participants. In the experiments presented in this paper, the set of 4 most selected images was used. The maximum size of visual summary generated by our algorithms was also set to 4 images.

- Selected by any participant.

Grouping similar images: In the ground truth collection process image similarity information was collected in the following way. A participant was allowed to specify that they did not pick a specific image, say X, for the summary, because it was similar to another image, say Y, that they did include in the summary. We use this information to group similar images. Result are presented that take this similarity into account. For example in the above scenario, if ground truth contains $\mathrm{Y}$, then the algorithm is scored identically if it selects $\mathrm{X}$ or $\mathrm{Y}$ as part of the visual summary.

Custom Metrics: In addition to presenting results for standard metrics (accuracy, precision, recall, F1-measure), we analyze the data to answer the following questions:

- What percentage of the summary images selected by the algorithm were not selected by any participant and not considered similar to any image selected by a participant?

- For what percentage of the images selected by 3 or more survey participants, the algorithm did not select the image or an image considered similar to it in the summary?

These question provide additional insight in the automated summary selection process given the fuzzy nature of the ground truth.

Perception of automated summaries: Finally, we present results on the perceived quality of the algorithm generated summary. This is an important measure as it is not uncommon to have a high quality visual summary that is significantly different from a summary provided by a human participant.

\section{RESULTS}

This work has developed a framework with a graph based algorithm to automatically select a set of images as a visual summary for a lecture video segment. For the purpose of evaluation, the framework was configured to select up to 4 images for the summary.

\subsection{Traditional Performance Metrics}

We evaluate our algorithm to automatically select a set of images with 4 different formulations

Top-4 Selected: The ground truth is composed of 4 images that were selected most often by the survey participants.

All Selected: The ground truth is composed of union of all images selected by the survey participants.

Top-4 Selected with Grouping: The images are first divided into groups based on any user specifying that one image is similar to another, as discussed in Section 6. The ground truth is composed of 4 groups whose members were selected most often by the survey participants.

All Selected with Grouping: The images are first divided into groups based on a user specifying that one image is similar to another, as discussed in Section 6. The ground truth is composed of all groups for which any image was selected by any survey participant.

Figure 9 shows algorithm performance with the formulations stated above. We make a few observations: i) The scores are significantly higher with grouping indicating the role played by user selected similar images, ii) precision is significantly higher but recall is lower when all participant choices are added to the ground truth, and iii) precision reaches a high 0.94 for "All Selected with Grouping" implying that most algorithm choices found some agreement with at least one survey participant.

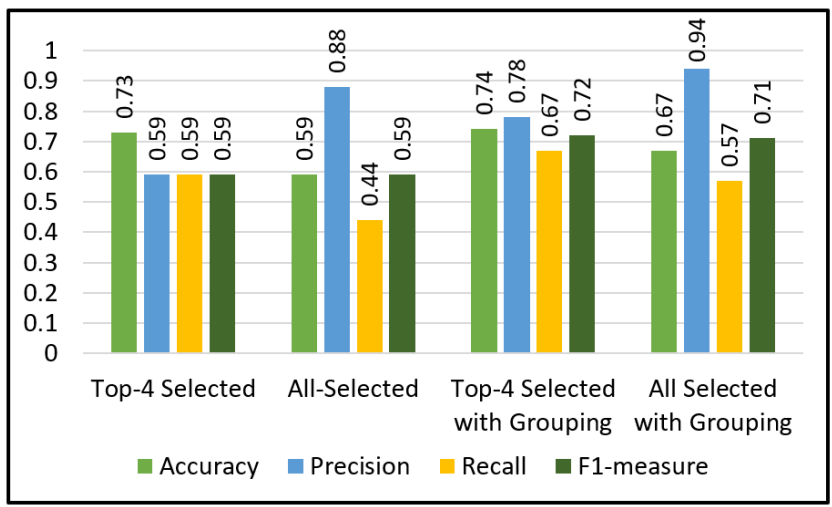

Figure 9: Performance of graph based visual summarization algorithm

\subsection{Comparison with Clustering}

We compare the results from our graph based algorithm with Kmedoid clustering algorithm [40]. We consider K-medoid to be a reasonable clustering algorithm in this scenario as it has been used in the context of image retrieval and face recognition [27, 47]. The 
results are presented in Figure 10. we note that our graph based algorithm yields slightly to significantly better performance on all metrics in the two scenarios that were compared.

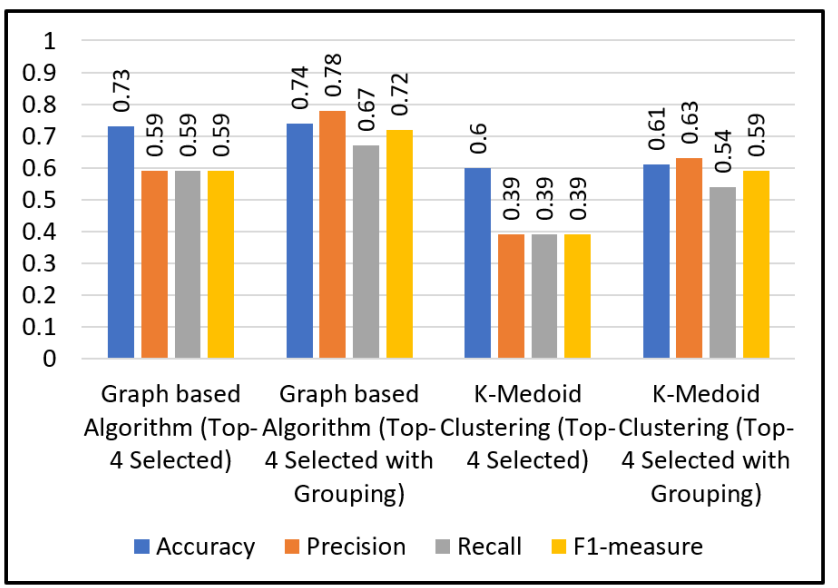

Figure 10: Performance of graph based algorithm vs Kmedoid clustering

\subsection{Custom Metrics}

We now address two specific questions that provide further insight into the ability of algorithms to correctly pick summary images.

- $12.5 \%$ of the summary images selected by the algorithm were not selected by any participant. Of these, only $1.88 \%$ were not selected by any participant and not considered similar to any image selected by a participant.

- $40 \%$ of the images selected by 3 or more participants were not selected by the algorithm. Around half of these, i.e., $20 \%$, were considered similar to an image selected by the algorithm.

In brief, the algorithm selected images were almost always considered relevant by at least one human, but top rated human images were not selected by the algorithm in several cases.

\subsection{User Perceptions}

User survey participants were provided with the algorithm selected visual summary and asked to rank its quality on a 4 point scale from "Poor" to "Very Good". Figure 11 plots the results. Figure 11 (a) shows that the users considered around $65 \%$ of segment summaries to be "Good" or "Very Good", and around 7.6\% of summaries to be "Poor". Figure 11 (b) plots the most positive review for a summary among all users who rated it. We observe that $85 \%$ of the algorithm generated summaries were rated to be "Very Good" by at least one user, and virtually all summaries were rated "Good" or "Very Good" by at least one user. Since evaluation of a summary is qualitative, it is an important result that at least one user was largely satisfied with an automatically generated summary for most segments.

We also surveyed the users for their level of familiarity with the content of the lecture videos they rated. Around $54.5 \%$ of the users considered themselves to be "Very Familiar", 25.2\% were "Familiar",

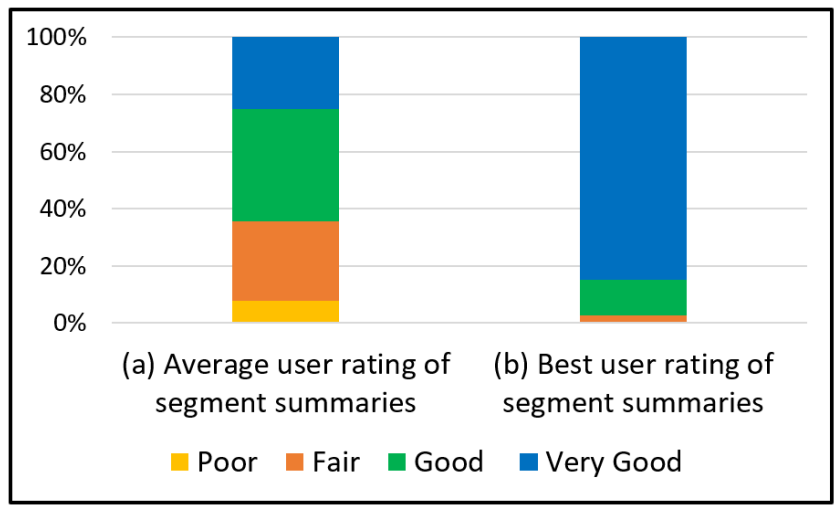

Figure 11: User perception of quality of algorithm generated summary

12.6\% were "Slightly Familiar" and 8.1\% were "Not at all Familiar". We observe that our efforts to match users with content matter they were familiar with were mostly successful. We verified that the small number of users with low familiarity do not meaningfully impact our results.

\section{CONCLUSIONS AND FUTURE WORK}

This paper presents a novel approach to use low-level image features to create a summary of visual contents extracted from a lecture video segment. The results are encouraging based on quantitative metrics as well as user perception of the quality of visual summaries. At the same time, there is also significant room for improvement.

Ongoing work is identifying and classifying the underlying causes of errors in visual summaries. Future work will focus on improving the accuracy and relevance of extracted summaries. Research directions under consideration include i) alternate image similarity measures, ii) analysis of high level semantic features, and iii) integrated text and image analysis. We also plan to substantially enhance the ground truth with additional surveys to be able to make more accurate assessments.

\section{ACKNOWLEDGMENTS}

The bulk of the Videopoints framework that we employed for evaluation was developed by a team led by Dr. Tayfun Tuna. Dr Thamar Solorio and Dr Ioannis Konstantinidis made numerous helpful suggestions on this project.

\section{REFERENCES}

[1] Gregory D. Abowd. 1999. Classroom 2000: An experiment with the instrumentation of a living educational environment. IBM Systems fournal 38, 4 (1999), 508âĂŞ-530. https://doi.org/10.1147/sj.384.0508

[2] Lecia Barker, Christopher L Hovey, Jaspal Subhlok, and Tayfun Tuna. 2014. Student Perceptions of Indexed, Searchable Videos of Faculty Lectures. In Proceedings of the 44th Annual Frontiers in Education Conference(FIE). IEEE, Madrid, Spain, $1-8$.

[3] L. Barker and J. Subhlok. 2012. Indexed Captioned Searchable Videos: Assessment Report. Technical Report UH-CS-11-07. University of Houston.

[4] Herbert Bay, Tinne Tuytelaars, and Luc Van Gool. 2006. SURF: Speeded Up Robust Features. In Computer Vision - ECCV 2006, Aleš Leonardis, Horst Bischof, and Axel Pinz (Eds.). Springer Berlin Heidelberg, Berlin, Heidelberg, 404-417.

[5] S. Belongie, J. Malik, and J. Puzicha. 2002. Shape matching and object recognition using shape contexts. IEEE Transactions on Pattern Analysis and Machine 
Intelligence 24, 4 (2002), 509-522.

[6] Marion Brandsteidl, Tanja Mayerhofer, Martina Seidl, and Christian Huemer 2012. Replacing Traditional Classroom Lectures with Lecture Videos: An Experience Report. In Proceedings of the 8th Edition of the EducatorsâĂZ Symposium (Innsbruck, Austria) (EduSymp â̆ $\breve{Z} 12$ ). Association for Computing Machinery, New York, NY, USA, 21âĂŞ27. https://doi.org/10.1145/2425936.2425940

[7] Jason A. Brotherton and Gregory D. Abowd. 2004. Lessons Learned from eClass: Assessing Automated Capture and Access in the Classroom. ACM Trans. Comput.Hum. Interact. 11, 2 (Jun 2004), 121-âĂŞ155. https://doi.org/10.1145/1005361. 1005362

[8] Martin C. Carlisle. 2010. Using You Tube to Enhance Student Class Preparation in an Introductory Java Course. In Proceedings of the 41st ACM Technical Symposium on Computer Science Education (Milwaukee, Wisconsin, USA) (SIGCSE â̆ÁZ10) Association for Computing Machinery, New York, NY, USA, 470âĂȘ474. https: //doi.org/10.1145/1734263.1734419

[9] Surendar Chandra. 2007. Lecture video capture for the masses. SIGCSE Bull. 39 3 (June 2007), 276-280. https://doi.org/10.1145/1269900.1268864

[10] Chekuri Choudary and Tiecheng Liu. 2007. Summarization of Visual Content in Instructional Videos. Multimedia, IEEE Transactions on 9 (12 2007), 1443 - 1455. https://doi.org/10.1109/TMM.2007.906602

[11] Anneliese Defranceschi and Marco Ronchetti. 2011. Video-lectures in a traditional mathematics course on iTunes U: Studentsâ̆ $\breve{Z}$ feedback. IEEE, Piestany, Slovakia, 219âĂŞ-224. https://doi.org/10.1109/ICL.2011.6059579

[12] Daniel DeMenthon, Vikrant Kobla, and David Doermann. 1998. Video Summarization by Curve Simplification. In Proceedings of the Sixth ACM International Conference on Multimedia (Bristol, United Kingdom) (MULTIMEDIA '98). ACM, New York, NY, USA, 211-218. https://doi.org/10.1145/290747.290773

[13] Rucha Deshpande, Tayfun Tuna, Jaspal Subhlok, and Lecia Barker. 2014. A Crowdsourcing Caption Editor for Educational Videos. In Proceedings of the 44th Annual Frontiers in Education Conference(FIE). IEEE, Madrid, Spain, 1-8.

[14] Paul E. Dickson. 2011. Using Undergraduate Teaching Assistants in a Small College Environment. ACM, New York, NY, USA, 75âĂS-80. https://doi.org/10.1145/ 1953163.1953187

[15] Paul E. Dickson, David I. Warshow, Alec C. Goebel, Colin C. Roache, and W. Richards Adrion. 2012. Student Reactions to Classroom Lecture Capture. ACM, New York, NY, USA, 144-âĂŞ149. https://doi.org/10.1145/2325296.2325334

[16] Ajay Divakaran, Kadir A. Peker, and Huifang Sun. 2001. Video summarization using motion descriptors. In Storage and Retrieval for Media Databases 2001, Minerva M. Yeung, Chung-Sheng Li, and Rainer W. Lienhart (Eds.), Vol. 4315 International Society for Optics and Photonics, SPIE, San Jose, CA, United States, 517 - 522. https://doi.org/10.1117/12.410963

[17] Echo360. 2020. Echo360. Echo360. https://echo360.com/

[18] L. Fei-Fei and P. Perona. 2005. A Bayesian Hierarchical Model for Learning Natural Scene Categories. CVPR 2 (2005), 524-531.

[19] J. Foertsch, G. Moses, J. Strikwerda, and M. Litzkow. 2002. Reversing the Lecture/Homework Paradigm Using eTEACHÂ̋̋ Web-based Streaming Video Software. Journal of Engineering Education 91 (2002), 267-274.

[20] K. Fujimura, K. Honda, and K. Uehara. 2002. Automatic video summarization by using color and utterance information. In Multimedia and Expo, 2002. ICME '02. Proceedings. 2002 IEEE International Conference on, Vol. 1. IEEE, Lausanne, Switzerland, Switzerland, 49-52 vol.1. https://doi.org/10.1109/ICME.2002.1035715

[21] Michael R Garey and David S Johnson. 1979. Computers and intractability. Vol. 174 freeman San Francisco, 41 Madison Avenue, 37th Fl. New York, NY, United States.

[22] Arun Hampapur, Terry E. Weymouth, and Ramesh Jain. 1995. Feature based digital video indexing. In Proceedings of the third IFIP WG2.6 working conference on Visual database systems 3 (VDB-3). Chapman \& Hall, Ltd., London, UK, UK, $115-141$.

[23] A. Hanger. 1995. A Survey of Technologies for Parsing and Indexing Digital Video. Technical Report TR-11-01-95. Boston University.

[24] C. Harris and M. Stephens. 1988. A Combined Corner and Edge Detector. In Proceedings of the Alvey Vision Conference 1 (1988), 147-151.

[25] Liwei He, Elizabeth Sanocki, Anoop Gupta, and Jonathan Grudin. 1999. AutoSummarization of Audio-Video Presentations. In Proceedings of the Seventh ACM International Conference on Multimedia (Part 1) (Orlando, Florida, USA) (MULTIMEDIA âĂŹ99). Association for Computing Machinery, New York, NY, USA, 489âĂŞ498. https://doi.org/10.1145/319463.319691

[26] Simon B. Heilesen. 2010. What is the academic efficacy of podcasting? Computer and Education 55, 3 (Nov 2010), 1063-âĂŞ1068. https://doi.org/10.1016/j.compedu. 2010.05.002

[27] Yushi Jing, Michele Covell, and Henry Rowley. 2010. Comparison of clustering approaches for summarizing large populations of images. In 2010 IEEE International Conference on Multimedia and Expo, ICME 2010. IEEE, Singapore, $1523-$ 1527. https://doi.org/10.1109/ICME.2010.5583276

[28] Amy N. B. Johnston, Helen Massa, and Thomas H. J. Burne. 2013. Digital lecture recording: A cautionary tale. Nurse Education in Practice 13, 1 (Jan 2013), 40. https://doi.org/10.1016/j.nepr.2012.07.004
[29] S. X. Ju, M. J. Black, S. Minneman, and D. Kimber. 1998. Summarization of videotaped presentations: automatic analysis of motion and gesture. IEEE Transactions on Circuits and Systems for Video Technology 8, 5 (Sep 1998), 686-696. https://doi.org/10.1109/76.718513

[30] Kaltura. 2020. Kaltura. Kaltura. https://corp.kaltura.com//

[31] Raga Shalini Koka. 2019. Automatic Keyword Detection for Text Summarization. Master's thesis. University of Houston, Houston, TX.

[32] Maureen J. Lage, Glenn J. Platt, and Michael Treglia. 2000. Inverting the Classroom: A Gateway to Creating an Inclusive Learning Environment. The fournal of Economic Education 31, 1 (Jan 2000), 30âĂŞ-43. https://doi.org/10.2307/1183338

[33] Jason W. Lancaster, Maureen L. McQueeney, and Jenny A. Van Amburgh. 2011. Online lecture delivery paired with in class problem-based learning âĂę Does it enhance student learning? Currents in Pharmacy Teaching and Learning 3, 1 (Jan 2011), 23-âĂS29. https://doi.org/10.1016/j.cptl.2010.10.008

[34] S. Lazebnik, C. Schmid, and J. Ponce. 2006. Beyond Bags of Features: Spatial Pyramid Matching for Recognizing Natural Scene Categories. CVPR 2 (2006), 2169-2178.

[35] T. Lindeberg. 1998. Feature Detection with Automatic Scale Selection. In International Journal of Computer Vision 30, 2 (1998), 79-116.

[36] T. Liu and C. Choudary. 2006. Content Extraction and Summarization of Instructional Videos. In 2006 International Conference on Image Processing. IEEE, Atlanta, GA, 149-152. https://doi.org/10.1109/ICIP.2006.312381

[37] Tiecheng Liu and J.R. Kender. 2002. Rule-based semantic summarization of instructional videos. In International Conference on Image Processing, Vol. 1. IEEE, NY, USA, I-601. https://doi.org/10.1109/ICIP.2002.1038095

[38] T. Liu and J.R. Kender. 2003. Semantic mosaic for indexing and compressing instructional videos. In International Conference on Image Processing, Vol. 1. IEEE, Barcelona, Spain, I - 921. https://doi.org/10.1109/ICIP.2003.1247114

[39] David Lowe. 2004. Distinctive Image Features from Scale-Invariant Keypoints. International fournal of Computer Vision 60 (11 2004), 91-110. https://doi.org/10. 1023/B\%3AVISI.0000029664.99615.94

[40] Tagaram Soni Madhulatha. 2011. Comparison between K-Means and K-Medoids Clustering Algorithms. In Advances in Computing and Information Technology, David C. Wyld, Michal Wozniak, Nabendu Chaki, Natarajan Meghanathan, and Dhinaharan Nagamalai (Eds.). Springer Berlin Heidelberg, Berlin, Heidelberg, 472-481.

[41] K. Mikolajczyk and C. Schmid. 2001. Indexing based on scale invariant interest points. In Proceedings Eighth IEEE International Conference on Computer Vision. ICCV 2001, Vol. 1. IEEE, Vancouver, BC, Canada, 525-531 vol.1.

[42] K. Mikolajczyk, T. Tuytelaars, C. Schmid, A. Zisserman, J. Matas, and L. Van Gool. 2006. A Comparison of Affine Region Detectors. International fournal of Computer Vision 65, 1âĂŞ2 (2006), 43-75.

[43] Hiroshi Mo, Fuminori Yamagishi, Ichiro Ide, Norio Katayama, Shin'ichi Satoh, and Masao Sakauchi. 2005. Key Image Extraction from a News Video Archive for Visualizing Its Semantic Structure. In Advances in Multimedia Information Processing - PCM 2004, Kiyoharu Aizawa, Yuichi Nakamura, and Shin'ichi Satoh (Eds.). Springer Berlin Heidelberg, Berlin, Heidelberg, 650-657.

[44] Blanche W. OBannon, Jennifer K. Lubke, Jeffrey L. Beard, and Virginia G. Britt. 2011. Using podcasts to replace lecture: Effects on student achievement. Computers and Education 57, 3 (Nov 2011), 1885-âĂŞ1892. https://doi.org/10.1016/j. compedu.2011.04.001

[45] Hamad Odhabi and Lynn Nicks-McCaleb. 2011. Video Recording Lectures: Student and Professor Perspectives. British fournal of Educational Technology 42 (2011), 327 - 336. https://doi.org/10.1111/j.1467-8535.2009.01011.x

[46] Panopto. 2020. Panopto. Panopto. https://www.panopto.com/

[47] Sarthak Parui and Anurag Mittal. 2014. Similarity-Invariant Sketch-Based Image Retrieval in Large Databases. In Computer Vision - ECCV 2014, David Fleet, Tomas Pajdla, Bernt Schiele, and Tinne Tuytelaars (Eds.). Springer International Publishing, Cham, 398-414.

[48] Swee Kit Alan Soong, Lay Kock Chan, and Christopher Cheers. 2006. Impact of video recorded lectures among students. Sydney University Press, Sydney, Australia, 789-âĂŞ793.

[49] S.D. Sorden and J.L. Ramirez-Romero. 2012. Collaborative Learning, Social Presence and Student Satisfaction in a Blended Learning Environment. IEEE, Rome, Italy, 129-âĂŞ133. https://doi.org/10.1109/ICALT.2012.114

[50] E. Tola, V. Lepetit, and P. Fua. 2008. A fast local descriptor for dense matching. In 2008 IEEE Conference on Computer Vision and Pattern Recognition. IEEE, Anchorage, AK, USA, 1-8.

[51] Ian N. Toppin. 2011. Video lecture capture (VLC) system: A comparison of student versus faculty perceptions. Education and Information Technologies 16, 4 (Dec 2011), 383âĂŞ-393. https://doi.org/10.1007/s10639-010-9140-x

[52] Tomoko Traphagan, John V. Kucsera, and Kyoko Kishi. 2010. Impact of class lecture webcasting on attendance and learning. Educational Technology Research and Development 58, 1 (Feb 2010), 19âĂŞ-37. https://doi.org/10.1007/s11423009-9128-7 
[53] Tayfun Tuna, Tuhin Dey, Jaspal Subhlok, and Leigh Leasure. 2017. Video Supported Flipped Classroom. In Proceedings of EdMedia: World Conference on Educational Media and Technology 2017, Joyce P. Johnston (Ed.). Association for the Advancement of Computing in Education (AACE), Washington, DC, 1159-1171. https://www.learntechlib.org/p/178432

[54] Tayfun Tuna, Mahima Joshi, Varun Varghese, Rucha Deshpande, Jaspal Subhlok, and Rakesh Verma. 2015. Topic based segmentation of classroom videos. In Proceedings of the 45th Annual Frontiers in Education Conference(FIE). IEEE, El Paso, Texas, 1-9.

[55] Tayfun Tuna, Jaspal Subhlok, Lecia Barker, Shishir Shah, Olin Johnson, and Christopher Hovey. 2017. Indexed Captioned Searchable Videos: A Learning Companion for STEM Coursework. Fournal of Science Education and Technology 26, 1 (2017), 82-99. https://doi.org/10.1007/s10956-016-9653-1

[56] Tayfun Tuna, Jaspal Subhlok, Lecia Barker, Varun Varghese, Olin Johnson, and Shishir Shah. 2012. Development and evaluation of indexed captioned searchable videos for STEM coursework.. In Proceedings of the 43rd SIGCSE Technical
Symposium on Computer Science Education. ACM, New York, NY, USA, 129-134.

[57] Tayfun Tuna, Jaspal Subhlok, and Shishir Shah. 2011. Indexing and keyword search to ease navigation in lecture videos. In Applied Imagery Pattern Recognition(AIPR). IEEE, Washington, DC, USA, 1-8

[58] Kuldeep Yadav, Ankit Gandhi, Arijit Biswas, Kundan Shrivastava, Saurabh Srivastava, and Om Deshmukh. 2016. ViZig: Anchor Points Based Non-Linear Navigation and Summarization in Educational Videos. In Proceedings of the 21st International Conference on Intelligent User Interfaces (Sonoma, California, USA) (IUI âĂŹ16). Association for Computing Machinery, New York, NY, USA, 407âĂŞ418. https://doi.org/10.1145/2856767.2856788

[59] Hongjiang Zhang, Chien Yong Low, and Stephen W. Smoliar. 1995. Video parsing and browsing using compressed data. Multimedia Tools and Applications 1, 1 (1995), 89-111. https://doi.org/10.1007/BF01261227 